MANAGEMENT

\section{Produkte statt Prozesse}

\author{
Claus Offermann
}

100 PS hat, vier Türen, unter fünf Liter Spritverbrauch auf 100 Kilometer und einen ordentlichen Kofferraum, dann lässt sich das Ergebnis oder der Grad der Zielerreichung nach der Produktion leicht begutachten. Wenn ein Patient beim Kratzen am Hinterkopf Schmerzen im Arm hat, ist das Produkt der Physiotherapeutin erstellt oder das Ziel ist erreicht, wenn der Patient nach der Behandlung seinen Arm schmerzfrei bewegen kann. Wenn ein Fahrgast in ein Taxi steigt und das Ziel »Bahnhof « angibt, dann ist das Produkt erstellt, wenn der Fahrgast am (richtigen) Bahnhof angekommen ist.

Gemeinsam ist diesen Produkten, dass es Vorgaben in Form von Zielen gibt, an denen sich die Ergebnisse messen lassen müssen. Das Produkt ist gelungen, wenn das Ziel erreicht ist.

\section{Materielle und immaterielle Produkte}

leistern Ergebnisse und nicht nur Prozesse erwartet werden,

- »sozial «, weil an diesen Ergebnissen der Kunde als Mitproduzent unmittelbar beteiligt ist,

- »immateriell«, weil die Ergebnisse nicht physisch vorhanden, also nicht lagerbar, nicht rückrufbar und nicht situationsunabhängig sind.

Dieser Perspektivenwechsel vom Prozess hin zum Produkt bringt den sozialen Produzenten einen Statusgewinn und den Kunden bessere Ergebnisse.

Ein Produkt ist ein Ergebnis oder die Erreichung eines Ziels, egal worin das Ziel besteht. Wenn das Ziel darin besteht, ein Auto zu bauen, das

Handlungen und Tätigkeiten, gleichgültig, ob sie materiell oder immateriell sind.

- Materielle Produkte sind relativ stabil, lassen sich zumindest eine bestimmte Zeit betrachten, berühren oder anderweitig sinnlich erfahren. Eine Vanillesoße gehört genauso dazu wie ein Mähdrescher. Die Haltbarkeit des Mähdreschers ist größer und er lässt sich auch mehrmals einsetzen, wohingegen die Vanillesoße sich nur einmal essen lässt. Beides sind jedoch materielle Produkte.

- Immaterielle Produkte sind eher flüchtig und lassen sich nicht lagern. Eine Taxifahrt zum Bahnhof ist ge-

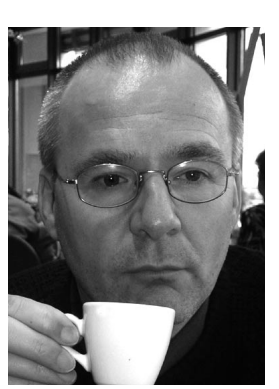

Dr. Claus Offermann ist Diplom-Verwaltungswissenschaftler und verfügt über langjährige Erfahrungen in der Bil-

dungs-, Sozial- und Gesundheitswirtschaft. Er ist als freiberuflicher Trainer und Berater in den Bereichen Soziales, Gesundheit und Bildung tätig. Zudem ist er als Zertifizierungsauditor bei ZertSozial GmbH in Stuttgart sowie im Beirat in der Internationalen Stiftung für Gesundheitsund Pflegenetze in Zürich und im Beirat der Akademie für integrative Bildung in Wien tätig. Internet http://www.qualitaetgestalten.de

nauso wenig lagerbar wie der Trost einer Pflegemitarbeiterin oder die Stimmung bei einem Fußballspiel.

Immaterielle Produkte werden häufig als »Dienstleistungen« bezeichnet. Dies ist im Grunde nicht korrekt: Eine Leistung ist ein Prozess und kein Ergebnis. Ein Prozess hat zwar auch Merkmale, aber er ist vom Ergebnis deutlich zu unterscheiden. Die Fahrt zum Bahnhof mit dem Taxi ist die Leistung. Das Produkt ist jedoch, dass der Fahrgast am Bahnhof ist. Möglicherweise ist der Taxifahrer ein netter Plauderer, der einem die Fahrt zum Bahnhof kurz erscheinen lässt. Das Plaudern ist der Prozess. Die Fahrt ist kurzweilig - das ist das Produkt.
Materielle Produkte lassen sich oftmals reparieren, wenn sie nicht gelingen oder zu Bruch gegangen sind. Immaterielle Produkte müssen neu in Angriff genommen werden. Der Taxifahrer bekommt ein neues Ziel, wenn er das angestrebte nicht erreicht hat. Ein Vorteil der immateriellen Produkte besteht sicherlich darin, dass sie nicht entsorgt werden müssen.

\section{Soziale Produkte}

Die Produzenten in der Sozialwirtschaft kennen häufig den Begriff des 


\section{Wie man Ziele und Produkte formuliert: Beispiel Kinderschutz}

Die derzeitige Praxis zeigt, dass in zahlreichen Auflagen des Jugendamtes beim Verdacht der Gefährdung des Kindeswohls schon Maßnahmen beschrieben werden (z. B. »Sie müssen jede Woche mit Michelle zum Kinderarzt!«), anstatt zunächst eine Zustandsbeschreibung zu formulieren, die das Kindeswohl sichert, also eine Beschreibung dessen, was alle im weiteren Verlauf geplanten und vereinbarten Maßnahmen bewirken müssen. Was zudem oftmals erfolgt ist, dass nicht in erster Linie die Verantwortung der Personensorgeberechtigten deutlich gemacht wird, sondern jegliches Handeln ausschließlich von ihnen persönlich gefordert wird (»Michelle muss von Ihnen täglich zum Kindergarten gebracht werden.«). Die Erreichung eines Zustandes, bei dem eine Gefährdung abgewendet ist, kann aber möglicherweise durch die Personensorgeberechtigten auch erreicht werden, indem diese ihre möglicherweise vorhandenen Netzwerke aktiviert.

Zudem werden in die Auflagen gern alle weiteren Aspekte mit hinein gepackt, die thematisch für die Jugendhilfe relevant sind. Motto: Wenn wir schon mal dabei sind, regeln wir gleich alles (selbst wenn bei den anderen Themen keine Gefährdung vorliegt). Formulierungen von Auflagen müssen also ein hohes Maß an Genauigkeit aufweisen und sich immer auf einen Sachstand innerhalb eines Gefährdungsbereiches (z. B. Aufsichtspflichtverletzung) beziehen.

Hinzu kommt, dass sich oftmals Auflagen nicht differenziert auf die Gefährdungsfaktoren einzelner Kinder beziehen, sondern pauschal auf alle Kinder der Familie. Es kann jedoch bei einem Kind einer Familie eine Gefährdung vorliegen, während diese Gefährdungsfaktoren für die Geschwisterkinder nicht zutreffen. Hat ein Kind in der Familie ein Ernährungsproblem, muss das noch lange nicht für die weiteren Kinder der Familie der Fall sein.

Des Weiteren werden Auflagen häufig abstrakt formuliert, manchmal in einer für die Personensorgeberechtigten unverständlichen Fachsprache: »Das Kind muss altersentsprechend ernährt sein « oder »Die Aufsichtspflicht ist zu gewährleisten.« Was heißt altersentsprechende Ernährung? Was heißt Aufsichtspflicht? Hier gilt es, ausgehend vom vorausgegangenen Sachstand der Kindeswohlgefährdung, festzuhalten, wie die konkrete Ernährung dieses Kindes auszusehen hat, damit die Gesundheit nicht gefährdet ist. Fachliche Vorgaben anderer Fachstellen und Professionen sind diesen zu überlassen, müssen aber Gegenstand der Auflage sein (»Sie als Vater müssen sicherstellen, dass Aische entsprechend des Ernährungsplans des Kinderarztes ernährt wird ; siehe Ernährungsplan in der Anlage.«).

Manchmal werden Auflagenformulierungen gewählt, die ausschließlich ein Unterlassen beinhalten: »Die Gefahrenquelle Toaster darf nicht mehr auf dem Esstisch stehen. «Stattdessen sollte eine Beschreibung vorliegen, die deutlich macht, wie ein Zustand auszusehen hat: »Sie als Mutter müssen ab sofort dafür sorgen, dass die Gefahrenquelle Toaster für Ihre Kinder unerreichbar ist. «Dies erleichtert den Personensorgeberechtigten eine Vorstellung davon zu bekommen, was konkret erreicht werden muss. Wenn Personensorgeberechtigte »nur « wissen, was sie nicht mehr tun dürfen oder wie etwas nicht mehr aussehen soll, wissen sie noch lange nicht, wie es stattdessen aussehen muss.

Sehr oft stoßen wir in Auflagen auf Optimal-Beschreibungen: »Die Küche ist jeden Tag geputzt, das Geschirr nach jedem Essen aufgeräumt. ¿Dann ist bei der Formulierung der Ausgangspunkt der Kindeswohlgefährdung nicht mehr erkennbar, nämlich verschimmelte herumliegende Lebensmittelreste, die von den Kindern gegessen wurden und immer wieder zu Durchfallerkrankung führten. Es wird ein aus der Sicht der Fachkraft wünschenswerter Zustand beschrieben. Bei der Formulierung von Auflagen gilt es die bürgerlich gefärbte Brille abzusetzen und ausschließlich die Gefährdungsaspekte zu fokussieren: »Sie als Mutter müssen $a b$ sofort dafür sorgen, dass verschimmelte Lebensmittelreste für Kinder unerreichbar sind und entsorgt werden. « Die anderen Themen sind entsprechend in den Leistungsbereich oder Graubereich einzuordnen und hier gilt es entsprechend entweder Ziele zu erarbeiten oder Aufträge zu formulieren.

Öfters kommt es vor, dass Auflagen nicht terminiert sind. Es wird nicht schriftlich festgehalten, ab wann (meist: ab sofort) und bis wann diese Auflagen gelten, als auch wann letztlich frühestens das Vertrauen in die Personensorgeberechtigten wieder hergestellt ist, also der damalige Sachverhalt sozusagen »verjährt« ist und man frühestens mit den Kontrollen aufhört.

Abschließend die dringende Empfehlung: Auflagen sollten allen Beteiligten in schriftlicher Form vorliegen. Nur dann ist ausreichend für alle dokumentiert, welche Auflage genau erteilt wurde.

Dr. Maria Lüttringhaus und Angelika Streich in den Blättern der Wohlfahrtspflege 4/2007 


\section{Wie man Ziele und Produkte formuliert: Beispiel Pflege}

Alle Handlungen einer Organisation sind zielgerichtet oder sollten sie zumindest sein. »Ziele zu finden und sie durchsichtig zu machen (...) ist eine Kunst «, schrieben Albrecht Müller-Schöll und Manfred Priebke* vor über dreißig Jahren in ihrem Standardwerk »Sozialmanagement (1976). Umso wichtiger sind die Ziele, wenn mit ihnen die Produkte einer Organisation beschrieben werden. Produkte werden auf unterschiedlichen Ebenen genannt, hier am Beispiel der Altenpflege.

Auf der Geschäftsführungsebene werden mitunter als Produkte »Wohlbefinden «, »Gepflegtsein «, »Sicherheit«, »Ruhe« oder »Freiheit« genannt. Ana$\log$ zu Müller-Schöll und Priebke sind es Produkte auf der Grundsatzebene. Je nach Klienten-, Patienten-, Kunden- oder Bewohnergruppe werden diese Produkte spezifischer formuliert.

Auf der Ebene des Pflegedienstes werden die Produkte zwar immer noch abstrakt, aber schon kundennäher formuliert. Pflegemodelle, vor allem »bedürfnisorientierte «, helfen bei der Orientierung der Produktgestaltung: »Die Bewohnerin kann ihre Bedürfnisse kommunizieren «, »Die Haut der Bewohnerin ist intakt «, »Die Bewohnerin ist angemessen beschäftigt « sind Produktbeschreibungen, die sich generell aus den Modellen und den Bedürfnislagen der Bewohnerinnen ableiten lassen. Analog zu Müller-Schöll und Priebke wird hier ein Produktrahmen vorgegeben. In Pflegekonzepten finden sich häufig in Form von Qualitätszielen die Rahmenangaben für immaterielle Pflegeprodukte.

Am schwierigsten ist die Ebene der Pflegesituationen, nämlich dort, wo die Produkte nicht nur als eine mehr oder weniger abstrakte Absichtserklärung ankommen, sondern als konkrete Ergebnisse. Ein Beispiel: »Die Bewohnerin ist auf ihrer rechten, auf der Lieblingsseite, stabil gelagert. Ihr Blick fällt auf das Marienbild an der Wand. Das Fenster ist schräg gestellt. « Das Produkt ist hier das Vorhandensein von bestimmten Merkmalen einer Situation. Die Situationen sind häufig vielschichtig und erfordern ein hohes Reflexions- und Gestaltungsvermögen des Produzenten. Analog zu Müller-Schöll und Priebke findet sich hier das Produkt als Ergebnis organisatorischen Handelns.

Es gibt auf dieser Ergebnisebene der Produkte Standardsituationen, in denen die Produkte immer gleich sind oder gleich sein sollten. Eine Injektion sollte so schmerzfrei wie möglich sein und den richtigen Teil des Körpers treffen. In Pflegestandards, die in Form von Zielen auch die Ergebnisse angeben, werden diese gleichförmigen Produkte und vor allem der Weg zu ihnen formuliert. Es gibt allerdings auch nicht formell standardisierte Situationen, bei denen die Ergebnisse oder Produkte mit der Patientin oder Bewohnerin mehr oder weniger ausgehandelt werden und Flexibilität bei der Produktgestaltung angesagt ist.

Ein Beispiel: Eine Pflegemitarbeiterin nimmt sich für die Abendtoilette folgende Pflegeziele vor: »Frau Karas sitzt stabil am Bettrand. Sie schlüpft mit meiner Hilfe selbstständig in ihre Pantoffeln. Sie geht an meinem Arm zum Waschbecken. Sie wäscht sich unter meiner Anleitung das Gesicht." So könnte das Produkt der Abendtoilette aussehen. Für diejenigen, die Schreibprobleme haben, sei gesagt: Auch wenn diese Ziele (oder Produkte) nicht aufgeschrieben werden, auf jeden Fall müssen sie gedacht werden und bei Bedarf auch mündlich benannt werden. Das Produkt der Pflegemitarbeiterin für Frau Karas wird jedoch an diesem Abend anders aussehen: Frau Karas fühlt sich elend und handlungsunfähig. Das Produkt ist nun: »Gesicht und Hände von Frau Karas sind ohne Mithilfe von Frau Karas erfrischt.«

Die immateriellen und materiellen Produkte unter Einbezug der Kundinnen neu zu formulieren und zu erbringen, das ist die Kunst der Berufe, die sich in Situationen bewähren müssen. Das soziale immaterielle Produkt entsteht auf dieser Ergebnisebene. Allerdings ist die Formulierung von Zielen und damit Produkten nicht gerade die Stärke der Berufe in der Sozial- und Gesundheitswirtschaft.

\section{Dr. Claus Offermann}

* Albrecht Müller-Schöll und Manfred Priepke: Sozialmanagement. Zur Förderung systematischen Entscheidens, Planens, Organisierens, Führens und Kontrollierens in Gruppen.

3. Auflage. Luchterhand Verlag, Neuwied 1992. 168 Seiten. 12,40 €. ISBN 978-3-472-01042-5. 
Produkts für ihren eigenen Wirkungsbereich nicht. Sie nehmen oft an, dass sie für die Tätigkeit vergütet werden und nicht für die Ergebnisse ihres Handelns. Es ist schwierig, wenn ein Produzent zwar weiß, was er tut oder tun soll, aber sein Produkt oder Ergebnis nicht oder nicht genau benennen kann.

Bei sozialen Produkten kommt das Produkt nur durch die Interaktion des Produzenten mit dem Kunden zustande. Oft bestimmt der Kunde durch den Grad seines Engagements über den Grad des Erfolgs, also den Grad der Zielerreichung mit. Alle sozialen immateriellen Produkte gehören in diese Kategorie. Pädagogen, Ärzte, Therapeuten, Pfleger sind in der Regel darauf angewiesen, dass der Kunde sich an der Erstellung des Produkts beteiligt.

Der Begriff des Sozialen ist hier nicht zu verwechseln mit dem Begriff des Guten, Edlen und Mildtätigen. Sozial bedeutet lediglich, dass eine Handlung auf einen anderen Menschen bezogen ist oder zusammen mit anderen durchgeführt wird.

Der Festlegung des sozialen Produkts geht häufig mehr oder weniger wahrnehmbar ein Aushandlungsprozess voraus. Ein Schulsozialarbeiter hat in der Einzelarbeit der Jugendhilfe möglicherweise eine aufwendige Diskussion darüber zu bestreiten, ob das Ziel, pünktlich zum Unterricht zu erscheinen, angemessen ist. Lässt sich der Jugendliche nicht auf dieses Ziel ein, dann wird der Sozialarbeiter möglicherweise ein Übergangsziel formulieren oder aus der Einzelarbeit aussteigen.

Weniger explizit sind die Aushandlungsprozesse über das Produkt bei stark gehandicapten Personen, die entweder nicht wagen zu widersprechen oder die nur schwach in der Lage sind, sich überhaupt zu äußern. Es kommt auf die Sensibilität des Produzenten an, verbale und nonverbale Signale wahrzunehmen und diese dann auch noch richtig zu deuten. Häufig ist in der Arbeit mit Menschen mit Behinderungen und mit dementen alten Menschen nur durch taktil erfasste Signale möglich zu erfahren, ob die Handlung, die zu einem bestimmten Ergebnis führen soll auch akzeptabel ist. Lieferanten, die nicht über die adäquaten Rezeptoren verfügen, tun sich schwer adäquate Produkte zu erstellen.
Hinzu kommt bei sozialen Produkten häufig, dass das Interesse des Produzenten des sozialen Produkts nicht nur darin besteht, für seine Tätigkeit bezahlt zu werden. Der Produzent hat häufig selbst Erwartungen an den Kunden. Der Arzt erwartet geradezu, dass der Patient sich aktiv an dem Genesungsprozess beteiligt, damit aus dem angestrebten Ziel auch ein gelungenes Produkt wird. Der Lehrer erwartet, dass seine Schüler lernen, damit sie einen guten Abschluss haben. Die Kunden akzeptieren meist diesen Anspruch ihrer Produzenten, ja sie fordern ihn teilweise sogar, sogar wenn sie selbst wenig oder nichts dazu beitragen, dass das Produkt gelingt. Manche Kunden leisten ihre Beiträge zwar, aber mitunter nur widerwillig.

Die soziale Kompetenz des Produzenten ist nicht nur darauf gerichtet, dass der Kunde das geplante Produkt reichten Zieles deutlich, dass der soziale Produzent seinen Teil der Vereinbarung erfüllt hat. Im Baumarkt kommt niemand auf die Idee, dass er noch einen Hammer umsonst bekommen kann, nur weil der Hammer grad da liegt. In der ambulanten Pflege ist dieses Argument in Form von »Sie könnten mir auch noch geschwind einen Kaffee, Frühstück machen, die Geschirrspülmaschine, Waschmaschine füllen, die Treppe runterputzen « für viele naheliegend.

- Auf der Ebene des Produkts ergeben sich unterschiedliche Handlungsalternativen für den Produzenten. Prozessstandards, wie bei Pflegestandards, lassen dem Produzenten wenig Spielraum, weil sie nicht nur die Ziele und Produkte, sondern auch die Prozesse konfektionieren. Produktorientierte Produzenten sind gegenüber den prozessorien-

\section{»Die Formulierung von Zielen gehört nicht zu den Stärken sozialer Berufe"}

akzeptiert, sondern der Kunde soll auch dazu motiviert werden, seinen Beitrag zur Erstellung des Produkts zu leisten. Das bedeutet mitunter, die Interessen des Kunden auch gegen dessen Widerstand zu vertreten. Je mächtiger der Lieferant in der Beziehung ist, desto eher wird ihm das gemeinsam ausgehandelte Produkt zusammen mit dem Kunden gelingen.

\section{Bedeutung und Folgen}

Der Nutzen des Perspektivenwechsels könnte sich auf mehreren Ebenen vollziehen:

- Auf der persönlichen Ebene des sozialen Produzenten kommt mit der Formulierung des Produkts ein machbares Ende in den Blick. Die »Ende-nie-Problematik « ist einer der Auslöser von Burn-out. Mit der Formulierung von erfüllbaren Zielen und der Bewertung der Zielerreichung wird auch dem Burn-out vorgebeugt.

- Auf der Ebene des Empfängers und Finanziers des sozialen Produkts wird mit der Feststellung des er- tierten Produzenten eher Tüftler, die sich etwas einfallen lassen (müssen), um ans Ziel zu kommen. Gebrauchsanweisungen können auch Laien befolgen.

- Auf der Ebene des Qualitätsmanagements wird in absehbarer Zeit zumindest die ISO 9001 nicht nur den Nachweis der Prozess-, sondern auch den Nachweis des Produktmanagements fordern.

- Auf der Ebene der Bewertung sind Produkte höherwertig als Prozesse. Selbst bei Ärzten sind diejenigen, die Produkte verkaufen besser bezahlt, als die Prozessverkäufer. Radiologen als Produzenten von Bildern und Gutachten verdienen deutlich mehr als Psychotherapeuten, die nach Zeit bezahlt werden.

Die Aufwertung der Ergebnisse der sogenannten Dienstleister ist schon lange fällig. Vielleicht trägt die Änderung der Perspektive von Prozessen zu Produkten dazu bei, den Status von sozialen immateriellen Produkten zu verbessern. Bedingung dafür ist allerdings, dass die sozialen Produzenten ihre Produkte formulieren können. 